VMB closer to CST I than controls (MJSD 0.21 vs 0.42 , $\mathrm{p}<0.01)$.

Conclusion Women on HC have more stable, Lactobacillusdominated VMB than controls. There is increased VMB stability after 3 months of HC use. Further assessment by HC type is currently being integrated into the analysis.

Disclosure No significant relationships.

\section{P592 MICROBIOTA CONCORDANCE BETWEEN MID-VAGINAL SWABS AND BOTH CLEAN- AND RANDOM-CATCH URINE SAMPLES}

${ }^{1}$ Courtney Robinson*, 'Johanna Holm, ${ }^{2}$ Sarah Brown, 'Jacques Ravel, ${ }^{3}$ Khalil Ghanem, ${ }^{4}$ Rebecca Brotman. ${ }^{1}$ University of Maryland, Institute for Genome Sciences, Baltimore, USA; ${ }^{2}$ University of Maryland, Baltimore, Institute for Genome Sciences, Baltimore, USA; ${ }^{3}$ Johns Hopkins, Infectious Diseases, Baltimore, USA; ${ }^{4}$ University of Maryland, Institute of Genome Sciences, Baltimore, USA

\subsection{6/sextrans-2019-sti.663}

Background While urine has successfully been used for STI testing, it has not been routinely used in urogenital microbiota studies. This work explores whether random-catch and/or clean-catch urine could be a proxy for assessing the vaginal microbiota.

Methods In two studies, urinary and vaginal microbiota from women ages 17-45 were compared for (1) 91 participants with paired mid-vaginal swabs and random catch urine samples and (2) 99 participants with paired mid-vaginal swabs and clean catch urine samples. Microbiota composition was characterized by amplicon sequencing of the V3-V4 regions of the 16S rRNA gene. Taxonomic classification was assigned based on SILVA and SpeciateIt. Community State Types (CST) were assigned using an algorithm trained on 13,000 well-characterized samples. CST I, II, III, and V were dominated by: Lactobacillus crispatus, L. gasseri, L. iners, and L. jensenii, respectively. CST IV-A, IV-B, and IV-C represented low-Lactobacillus states. Similarity of paired urine and vaginal samples was measured at the CST-level by kappa statistics and the population-level with the Yue-Clayton theta indices.

Results We obtained 12 and 7.8 million sequences from urine and vaginal samples, respectively. At the CST-level, randomcatch and clean-catch urines were $82.4 \%$ and $81.8 \%$ concordant with paired mid-vaginal swabs, respectively. Substantial agreement was observed between urine and paired vaginal specimen $\left(\mathrm{K}_{\text {random-catch }}=0.770\right.$ and $\left.\mathrm{K}_{\text {clean-catch }}=0.743\right)$. At a population-level, average similarity of random- and clean-catch samples to paired vaginal samples indicated a high degree of similarity $(\theta=0.7496$ and 0.7565 , respectively). Comparison of the distributions of random-catch and clean-catch $\theta$ similarity scores showed no differences $(p=0.86)$.

Conclusion Bacterial compositions of random catch and clean catch urine samples showed substantial agreement to paired mid-vaginal samples assessed by CST- and community-level analyses. Random and clean catch urine samples could potentially be used as a proxy for vaginal microbiota in studies assessing the urogenital microbiota.

Disclosure No significant relationships.

\section{P593 A CROSS-SECTIONAL STUDY OF BIRTH MODE AND VAGINAL MICROBIOTA IN REPRODUCTIVE-AGE WOMEN}

${ }^{1}$ Christina Stennett, ${ }^{2}$ Typhanye Dyer, ${ }^{3}$ Xin He, ${ }^{4}$ Jacques Ravel, ${ }^{5}$ Khalil Ghanem, ${ }^{6}$ Rebecca Brotman*. 'University of Medicine School of Medicine, Institute for Genome Sciences, Baltimore, USA; ${ }^{2}$ University of Maryland, Epidemiology and Biostatistics, College Park, USA; ${ }^{3}$ University of Maryland, College Park, Epidemiology and Biostatistics, College Park, USA; ${ }^{4}$ University of Maryland School of Medicine, Institute of Genome Sciences, Baltimore, USA; ${ }^{5}$ Johns Hopkins, Infectious Diseases, Baltimore, USA; ${ }^{6}$ University of Maryland, Institute of Genome Sciences, Baltimore, USA

\subsection{6/sextrans-2019-sti.664}

Background Recent data suggests that birth mode (Cesarean section [C-section] versus vaginal delivery) is an important seeding event in the initial colonization of the human microbiome and is associated with long-term health. We sought to determine the association between $\mathrm{C}$-section delivery and vaginal microbiota in adulthood.

Methods We re-contacted 144 adult women from two concluded studies. In a phone survey, women reported their birth mode, obesity, breastfeeding, and age at menarche. Vaginal microbiota was characterized on a single baseline sample by amplicon sequencing of the V3-V4 hypervariable regions of the 16S rRNA gene and clustered into community state types (CSTs). We evaluated the association between birth mode and low versus high relative abundance of Lactobacillus spp. in logistic regression models controlling for body mass index, a significant confounder in this study.

Results 19\% $(\mathrm{n}=27)$ reported C-section delivery. Overall, Csection was non-significantly associated with increased odds of a low-Lactobacillus CST (aOR=1.22, 95\% CI: 0.45, 3.32). Because the two archived studies had different participant characteristics and inclusion criteria (interaction $p=0.048$ ), we stratified the analysis by study site. In the study with larger sample size $(n=88)$, women born via C-section had 3-fold higher odds of having low-Lactobacillus vaginal communities compared to vaginally-delivered women $(\mathrm{aOR}=3.55, \mathrm{p}=0.06$, 95\% CI: $0.97,13.02)$. No association was found in the smaller study $(\mathrm{n}=56, \mathrm{aOR}=0.19, \mathrm{p}=0.14,95 \% \quad \mathrm{CI}: 0.02$, 1.71).

Conclusion This cross-sectional study suggests a possible association between C-section and increased odds of a low-Lactobacillus vaginal microbiota in adulthood. However, this analysis is limited by relatively small sample size and lack of comparability in participant age, personal hygiene behaviors, and other characteristics between the study sites. Future longitudinal studies could better account for expected fluctuations in CST and may also explore confounders including behavioral factors and socioeconomic status known to be associated with both delivery mode and vaginal microbiota.

Disclosure No significant relationships. 